\title{
Optimal Routing, Link Scheduling and Power Control in Multi-hop Wireless Networks
}

\author{
R. L. Cruz and Arvind V. Santhanam \\ Department of Electrical \& Computer Engineering \\ University of California, San Diego \\ La Jolla, CA 92093-0407 \\ cruz@ece.ucsd.edu, arvind@cwc.ucsd.edu
}

\begin{abstract}
In this paper, we study the problem of joint routing, link scheduling and power control to support high data rates for broadband wireless multi-hop networks. We first address the problem of finding an optimal link scheduling and power control policy that minimizes the total average transmission power in the wireless multi-hop network, subject to given constraints regarding the minimum average data rate per link, as well as peak transmission power constraints per node. Multi-access signal interference is explicitly modeled. We use a duality approach whereby, as a byproduct of finding the optimal policy, we find the sensitivity of the minimal total average power with respect to the average data rate for each link. Since the minimal total average power is a convex function of the required minimum average data rates, shortest path algorithms with the link weights set to the link sensitivities can be used to guide the search for a globally optimum routing. We present a few simple examples that show our algorithm can find policies that support data rates that are not possible with conventional approaches. Moreover, we find that optimum allocations do not necessarily route traffic over minimum energy paths.
\end{abstract}

Index Terms-Wireless Networks, Multi-hop Networks, Routing, Scheduling, Power Control.

\section{INTRODUCTION AND MODEL}

Broadband wireless networks today are capable of supporting high data rates. Minimizing the total power in such systems is of paramount importance not just to increase its own operational lifetime in the case of battery powered devices, but also to coexist symbiotically with other systems which share the same frequency spectrum. For instance, 802.11 LANs and Bluetooth networks share the same unlicensed band and can mutually benefit by limiting the power of their respective signal transmissions.

Most currently deployed networks that support high data rates use only a "single hop" for wireless communication between a mobile user and an access point that is connected to the wired infrastructure. However, the use of multiple hops to transport data has been shown to enhance network capacity [10] [9] [17], and may be necessary due to cabling limitations in many environments.

Consider a network of base-stations interconnected to each other through wireless links. Each base-station serves as an ingress or egress for the aggregate of traffic associated with the mobile users in its domain, and routes its data through other base stations via multiple wireless hops to an access point connected to a wired infrastructure. In this paper, we focus on the transport of such aggregated traffic between base stations. We present algorithms to compute the optimal routing, link scheduling and power control policy that consumes minimum total average power. While current approaches tend to de-couple routing, link scheduling and power control from each other, we take an integrated approach.

Suppose there are $N$ stationary nodes, labeled by the integers $1,2, \ldots, N$, which correspond to the base stations discussed in the previous paragraph. A set $\mathcal{E}$ of $L_{\mathcal{E}}=|\mathcal{E}|$ transmission links, among the possible $N(N-1)$ links between nodes, constitutes a network topology. For now, assume that the transmissions on all of these links occur concurrently.

For a given link $l=(i, j)$, the transmitter node $i$ uses a signal power $P(l)$. The path "gain" from node $i$ to node $j$ is given by $G(i, j)$, and models the effects of signal attenuation due to distance, channel fading and shadowing, as well as antenna gain patterns. We assume that the path gains $G(i, j)$ are constant. The transmitting and receiving nodes of link $l$ are denoted by $T(l)$ and $R(l)$ respectively. The received signal power at node $R(l)$ from the transmitter $T(l)$ thus given by $P(l) G(T(l), R(l))$. However, signals emanating from other transmitters appear to the receiver $R(l)$ as interference, and there is ambient thermal noise to contend with as well. The signal to interference and noise ratio (SIR ) for link $l$ is defined as

$$
\gamma(l)=\frac{G(T(l), R(l)) P(l)}{\sum_{k: k \neq l} P(k) G(T(k), R(l))+n_{R(l)}},
$$

where $n_{j}$ is the ambient noise power at node $j$.

The capacity of the single wireless channel embodied by link $l$ can be modeled as a function of the SIR, $\gamma(l)$. For instance, the Shannon capacity of a link $l$ over a frequency bandwidth $W$ is $W \log _{2}(1+\gamma(l))$, assuming Gaussian noise and interference. Throughout this paper, we assume that the achieved data rate on link $l, X(l)$, is a linear function of the SIR, $\gamma(l)$, and the total available bandwidth $W$. This is a reasonable approximation to the Shannon capacity at small values of SIR, which for example is the case in CDMA networks where all links share a common frequency band and have at least a moderate processing gain. Our model could easily encompass the case where the links 
may use different frequency bands, and in this case the path gains incorporate crosstalk due to imperfect bandpass filters. However, for concreteness throughout this paper, we shall assume all links share the same frequency band of width $W$. Assuming the Gaussian approximation [14] to compute the biterror-rate (BER), the data rate of a link $l$ with a tolerable BER of $10^{-q}$ and using BPSK modulation is given by

$$
X(l)=W^{\prime} \gamma(l) \quad \text { where } \quad W^{\prime}=\frac{W}{2 q \log _{2} 10} .
$$

Suppose that the desired data rate on each link $l$ is $C(l)$ bits per second, i.e.

$$
X(l) \geq C(l), l \in L_{\mathcal{E}} .
$$

Using (1) and (2), we can rewrite (3) in matrix form as

$$
\mathbf{P} \geq \mathbf{F P}+\mathbf{b}
$$

where $\mathbf{P}=\left(P(1), \ldots, P\left(L_{\mathcal{E}}\right)\right)^{T}$,

$$
b(l)=\frac{C(l) n_{R(l)}}{G(R(l), T(l)) W^{\prime}},
$$

$\mathbf{b}=\left(b(1), \ldots, b\left(L_{\mathcal{E}}\right)\right)^{T}$, and $\mathbf{F}$ is the $L_{\mathcal{E}} \times L_{\mathcal{E}}$ matrix with entries

$$
F(l, k)= \begin{cases}\frac{G(R(l), T(k)) C(l)}{G(R(l), T(l)) W^{\prime}} & , \text { if } k \neq l \\ 0 & \text {, if } k=l .\end{cases}
$$

If $\mathbf{F}$ is a stable matrix, i.e. all eigenvalues of $\mathbf{F}$ are strictly inside the unit circle, then $(\mathbf{I}-\mathbf{F})^{-1}$ exists and has nonnegative elements. Thus, in this case, we can rearrange (4) and premultiply the resulting matrix inequality by $(\mathbf{I}-\mathbf{F})^{-1}$ to obtain

$$
\mathbf{P} \geq(\mathbf{I}-\mathbf{F})^{-1} \mathbf{b}
$$

Hence, we can view $(\mathbf{I}-\mathbf{F})^{-1} \mathbf{b}$ as the minimal power vector that supports the network topology defined by links $\mathcal{E}$ and their associated required data rates. Foschini and Miljanic [8] proposed a simple distributed algorithm to compute the minimum power vector in a cellular network context. Subsequently, Bambos, Chen, and Pottie [1] considered a distributed link admission control algorithm for the case of multi-hop networks, in the framework of [8].

If the eigenvalues of $\mathbf{F}$ are on or outside the unit circle, there does not exist any power vector $\mathbf{P}$ which supports the required data rates. If the eigenvalues of $F$ are inside the unit circle but close to the boundary, the minimal power vector $(\mathbf{I}-\mathbf{F})^{-1} \mathbf{b}$ will be very large. Even if the eigenvalues of $\mathbf{F}$ are well inside the unit circle, using the minimal power vector indicated in (5) may be inefficient. This is illustrated in the following example.

Example: Consider a square network of 4 nodes and 2 links $\{(1,2),(3,4)\}$. Suppose the relevant path gain values are $G(1,2)=G(3,4)=G_{0}$ and $G(1,4)=G(3,2)=$ $G_{0} / 2$, corresponding to omnidirectional antennas and inverse square law propagation loss. Let the required data rates be $X(\{1,2\})=X(\{3,4\})=\frac{W^{\prime}}{2}$, and let the ambient noise $n_{0}$ be the same at both the receivers, specifically $n_{0}=G_{0}$. In this case, it is readily verified that $(\mathbf{I}-\mathbf{F})^{-1} \mathbf{b}=(0.67,0.67)^{T}$, i.e. each transmitter is required to continuously transmit at a power of 0.67 Watt. On the other hand, if instead the transmitters take turns transmitting at a power of 1 Watt, such that only one transmitter is active at any time, the peak data rate that can be achieved is twice that of the data rate achieved previously, due to a lack of interference. Thus, if the transmitters are active for $50 \%$ of the time, the same average data rate of $\frac{W^{\prime}}{2}$ is achieved, but this consumes an average power of only 0.5 Watt, which is $33 \%$ less than before.

This example suggests that it can be inefficient to operate with all links in a wireless network active concurrently. Indeed, in most wireless networks, links operate in a half-duplex mode to limit interference. We can essentially eliminate interference by scheduling only links which are very far apart, as is proposed for example in [7][11]. However, in order to achieve high data rates, this may require unacceptably high peak transmission power. Constraints on peak transmission power may necessitate the use of concurrent transmissions in this case.

Recently, Elbatt and Ephremides [6] proposed a scheduling and power control strategy that selects candidate subsets of concurrently active links, and applies the power control algorithm in [8] in order to find the minimal power vector $(\mathbf{I}-\mathbf{F})^{-1} \mathbf{b}$ for each candidate subset. The objective of their optimization problem is to maximize network throughput by supporting as many links as possible with a given fixed SIR, while remaining energy efficient. They propose a heuristic to identify large subsets of concurrently active links that can be efficiently supported, and evaluate the throughput of the heuristic via simulation for a uniform traffic demand on links.

In this paper, we propose a systematic optimization for a problem formulation that is motivated by similar objectives. In particular, we seek to find subsets of simultaneously active links, herein called transmission modes, as well as the associated transmission powers, in order to minimize the total average transmission power expended across the network. The constraints of this optimization problem are that each link in the network must have an average data rate no less than a given prescribed value, and a peak transmission power for each node. This problem statement is formalized in Section II, and a duality approach for finding the optimal scheduling and power control policy is presented.

The required minimum average data rates on each link are ultimately determined by the average rates at which traffic is generated by users and the routing algorithm that is used. In wireless networks it is possible to reconfigure the data rates of the links on a fast time scale in response to changing traffic and channel conditions. In this paper, we shall also consider the problem of routing, and hence determination of the required data rates on each link, for a given traffic demand rate matrix. We shall address this in Section III, exploiting the duality approach explained in Section II.

After presenting the details of our approach, we discuss several illustrative examples in Section IV. Qualitatively, we find that in high ambient noise regimes, a large number of 
links in close geographic proximity are active simultaneously in optimal policies. In addition, we find that route diversity allows nodes to split traffic over multiple paths and support higher throughputs than could otherwise be achieved. This is somewhat surprising since all links share a common bandwidth. However, pushing the data rates to the maximum possible limit comes at price: As the data rates increase, the network tends to become less energy efficient. We discuss the applicability of our proposed routing, scheduling and power control algorithms to minimize the sum total of average transmitter and receiver power subject to a minimum average rate per link and peak power per node in Section V. We conclude the paper with some closing remarks in Section VI.

\section{Optimal Scheduling AND Power Control}

In this section, we formally specify the optimization problem discussed in the previous section, and present a duality approach for solving it. We then discuss an approach to limit computational complexity. We also briefly discuss an hierarchical approach that we developed recently in [5] that allows our link scheduling and power control algorithms presented in this paper, to scale for multihop networks with a large number of links.

For simplicity of exposition, we divide time into slots, each of equal duration and indexed by the positive integers. Transmissions begin and end on slot boundaries. Generalizing the notation introduced earlier, let $X_{m}(l)$ and $P_{m}(l)$ be the data rate for link $l$ in slot $m$, and transmission power for the transmitter $T(l)$ for link $l$ in slot $m$, respectively. Let $\vec{P}_{m}=\left(P_{m}(1), P_{m}(2), \ldots, P_{m}\left(L_{\mathcal{E}}\right)\right)$ be the network power vector for slot $m$. Let $P^{\max }(i)$ be the maximum transmission power for node $i$. Let $\mathcal{E}(i)$ be the links in $\mathcal{E}$ that originate at node $i$.

Each node must conform to the peak transmission power constraint in every slot:

$$
\begin{aligned}
& 0 \leq \sum_{l \in \mathcal{E}(i)} P_{m}(l) \leq P^{\max }(i) \text { and } \\
& 0 \leq P_{m}(l), \text { for all } m \geq 1 .
\end{aligned}
$$

Using (2) and (1), the achieved data rate for link $l$ in slot $m$ is

$$
X_{m}(l)=W^{\prime}\left(\frac{G(T(l), R(l)) P_{m}(l)}{\sum_{k: k \neq l} G(T(k), R(l)) P_{m}(k)+n_{R(l)}}\right) .
$$

The long-term average rate of link $l$ is then defined as

$$
X^{a v g}(l)=\liminf _{m \rightarrow \infty} \frac{1}{m} \sum_{k=1}^{m} X_{k}(l)
$$

For each link $l$, let $C(l)$ be a given minimum acceptable average data rate, i.e. we must have

$$
X^{a v g}(l) \geq C(l), \text { for all } l \in \mathcal{E} .
$$

Define the required minimum average rate vector as $\vec{C}=$ $\left(C(1), C(2), \ldots, C\left(L_{\mathcal{E}}\right)\right)$.
The average power consumed by the transmitter for link $l$, $P^{a v g}(l)$, is

$$
P^{\text {avg }}(l)=\limsup _{m \rightarrow \infty} \frac{1}{m} \sum_{k=1}^{m} P_{k}(l) .
$$

Define the average network power vector as $\vec{P}^{a v g}=$ $\left(P^{a v g}(1), P^{a v g}(2), \ldots, P^{a v g}\left(L_{\mathcal{E}}\right)\right)$.

There may or may not exist a sequence of network power vectors $\vec{P}_{1}, \vec{P}_{2}, \ldots$ that satisfy (6) and (7). If there does exist a sequence of such network power vectors, our aim is to minimize a linear function of $\vec{P}^{a v g}$. An example of such a linear function is simply the total average power. We will consider the slightly more general cost function

$$
h\left(\vec{P}^{a v g}\right)=\sum_{l \in \mathcal{E}} \alpha(l) P^{a v g}(l),
$$

where $\alpha(l)$ is a positive weight. For example, $h\left(\vec{P}^{a v g}\right)$ might equal the average power incident at a given point in space, or be equal to the total average power emanating from a closed boundary surrounding the network. Alternatively, $\alpha(l)$ might model the cost of a battery that drives transmitter $T(l)$.

\section{A. Primal Problem}

One of the main aims of this paper is to present an approach to compute a schedule of network power vectors $\vec{P}_{1}, \vec{P}_{2}, \ldots$, satisfing the peak power constraints (6) and the minimum average rate constraints $(7)$, such that $h\left(\vec{P}^{a v g}\right)$ is minimized. This primal problem is succinctly stated as

$$
\min \left\{h\left(\vec{P}^{a v g}\right)\right\} \text { subject to }(6) \text { and }(7) .
$$

Note this optimization involves choosing optimal power levels in each slot for each transmitter. Using a duality approach below, we reduce the problem to a convex optimization problem over a single slot.

\section{B. Duality Approach}

Let the value of the optimal cost in the problem (9) as a function of $\vec{C}$ be denoted by $H(\vec{C})$. If for a given value of $\vec{C}$, no schedule of network power vectors exists satisfying (6) and (7), define $H(\vec{C})=+\infty$.

Note that $H(\vec{C})$ is a convex function of the vector $\vec{C}$. To see this, suppose that $\vec{C}=\lambda \vec{C}_{1}+(1-\lambda) \vec{C}_{2}$, where $0<\lambda<1$. Let $\pi_{i}$ be an optimal policy that supports the average date rates specified by $\vec{C}_{i}$, i.e it achieves minimum cost $H\left(\vec{C}_{i}\right)$. Roughly speaking, we can create a new policy $\pi$ by "time sharing" between the policies $\pi_{1}$ and $\pi_{2}$, such that policy $\pi_{1}$ is used a fraction $\lambda$ of the time and policy $\pi_{2}$ is used a fraction $1-\lambda$ of the time. The policy $\pi$ supports average data rate rates that are at least as large as $\vec{C}$. Furthermore, by the linearity of $h(\cdot)$, the cost of policy $\pi$ is $\lambda H\left(\vec{C}_{1}\right)+(1-\lambda) H\left(\vec{C}_{2}\right)$. By definition of $H(\vec{C})$, the cost of policy $\pi$ is at least $H(\vec{C})$. Thus we have $H(\vec{C}) \leq \lambda H\left(\vec{C}_{1}\right)+(1-\lambda) H\left(\vec{C}_{2}\right)$. 
Define a set of dual variables $\vec{\beta}=\left\{\beta(1), \beta(2), \ldots, \beta\left(L_{\mathcal{E}}\right)\right\}$ for each link. Define a "potential" function $V$ according to

$$
V(\vec{P}, \vec{C}, \vec{X})=h(\vec{P})+\sum_{l \in \mathcal{E}} \beta(l)[C(l)-X(l)]
$$

The dual objective function is defined as

$$
g(\vec{\beta})=\min \left\{V\left(\vec{P}^{a v g}, \vec{C}, \vec{X}^{a v g}\right)\right\} \text { subject to }(6) .
$$

Note the absence of the minimum rate constraints (7) in the definition of $g(\vec{\beta})$. For any non-negative vector $\vec{\beta}$, using (7), note that

$$
\begin{aligned}
H(\vec{C}) & \geq \min \left\{V\left(\vec{P}^{a v g}, \vec{C}, \vec{X}^{a v g}\right)\right\}, \text { subject to (6) and (7) } \\
& \geq \min \left\{V\left(\vec{P}^{a v g}, \vec{C}, \vec{X}^{a v g}\right)\right\}, \text { subject to (6) } \\
& =g(\vec{\beta})
\end{aligned}
$$

Thus, for any non-negative vector $\vec{\beta}$, the minimum value of the objective function in the optimization problem (9) is lower bounded by $g(\vec{\beta})$. This observation leads to the dual optimization problem,

$$
\max \{g(\vec{\beta}): \vec{\beta} \geq \overrightarrow{0}\} .
$$

A geometric interpretation of the inequality (10) can be obtained by considering the surface of $H(\cdot)$ in $\left(L_{\mathcal{E}}+1\right)$ dimensional Euclidean space. In particular, $H(\vec{C})$ is lower bounded by considering a supporting hyperplane that is normal to the vector $(\vec{\beta}, 1)$. Since $H(\cdot)$ is convex, there exists a supporting hyperplane which passes through the point $(\vec{C}, H(\vec{C}))$. This is simply the classical argument used to prove that there is no "duality gap," [2] i.e.

$$
\begin{aligned}
H(\vec{C}) & =\max \{g(\vec{\beta}): \vec{\beta} \geq \overrightarrow{0}\} \\
& =g\left(\vec{\beta}^{*}\right) .
\end{aligned}
$$

Here, the supporting hyperplane passing through the point $(\vec{C}, H(\vec{C}))$ is normal to the vector $\left(\vec{\beta}^{*}, 1\right)$. From this geometric interpretation, it can be seen that the components of the optimal dual variable vector $\vec{\beta}^{*}$ represent a sensitivity of the optimal cost with respect to a perturbation in the minimum average data rate for a link, i.e.

$$
\beta^{*}(l)=\frac{\partial H(\vec{C})}{\partial C(l)} .
$$

Computation of $g(\vec{\beta})$ involves optimizing over all schedules of network power vectors satisfying the peak power constraint in every slot. However, since the potential function $V(\vec{P}, \vec{C}, \vec{X})$ is linear in $\vec{P}, \vec{C}$, and $\vec{X}$, it follows that $g(\vec{\beta})$ can be computed by an optimization over a single slot, i.e.

$$
g(\vec{\beta})=\min \left\{V(\vec{P}, \vec{C}, \vec{X}): \vec{P} \in S_{P}\right\},
$$

where $\vec{X}=\vec{X}(\vec{P})$ is defined as in (2) and (1), and $S_{P}$ is defined as the polyhedral set $S_{P}=\left\{\vec{P}: \sum_{l \in \mathcal{E}(i)} P(l) \leq\right.$ $P^{\max }(i)$ for all $i$ and $0 \leq P(l)$ for all $\left.l \in \mathcal{E}\right\}$. Let $M$ be the number of extreme points of $S_{P}$, and let $\vec{P}_{m}^{e x t}, m=1,2, \ldots M$ denote the extreme points of $S_{P}$. By definition, each point in $S_{P}$ can be represented as a convex combination of $\vec{P}_{m}^{e x t}$, $m=1,2, \ldots M$. An upper bound on $M$ is $2^{L_{\mathcal{E}}}$. If $M_{i}$ is the number of links emanating from node $i$, then it can be shown that $M=\Pi_{i=1}^{N}\left(1+M_{i}\right)$.

Even though it can be verified that $V(\vec{P}, \vec{C}, \vec{X}(\vec{P}))$ is not a concave function of the vector $\vec{P}$, it is easy to show by taking derivatives that $V(\vec{P}, \vec{C}, \vec{X}(\vec{P}))$ is a concave function of $P(l)$ for each $l \in \mathcal{E}$. A standard convexity argument then shows that

$$
g(\vec{\beta})=\min \left\{V\left(\vec{P}_{m}^{e x t}, \vec{C}, \vec{X}\left(\vec{P}_{m}^{e x t}\right)\right): 1 \leq m \leq M\right\} .
$$

Thus, the complexity of computing $g(\vec{\beta})$ is $O(M)$. In fact, note that $V\left(\vec{P}_{m}^{e x t}, \vec{C}, \vec{X}\left(\vec{P}_{m}^{e x t}\right)\right)$ is an affine function of $\vec{\beta}$, which implies that the dual objective function $g(\vec{\beta})$ is the pointwise minimum of $M$ affine functions, i.e.

$$
g(\vec{\beta})=\min _{1 \leq m \leq M}\left\{\mathbf{a}_{\mathbf{m}}^{\prime} \vec{\beta}+b_{m}\right\} .
$$

We have developed an iterative ascent algorithm to solve the dual problem (11). Exploiting the piecewise linearity of $g(\vec{\beta})$, our algorithm converges in a finite number of iterations. Alternately, by introducing a slack variable, the dual problem (11) can be reduced to a linear program and be efficiently solved using interior point methods.

If the required minimum rate vector $\vec{C}$ is infeasible, i.e. $H(\vec{C})=+\infty$, it can be seen that $g(\vec{\beta})$ is unbounded from above over the set of all $\vec{\beta} \geq 0$. If $\vec{C}$ is feasible, it is easy to see that

$$
H(\vec{C}) \leq \sum_{i=1}^{N} P^{\max }(i) .
$$

Thus we can safely declare $\vec{C}$ infeasible if we find a value of $\vec{\beta}$ such that $g(\vec{\beta})>\sum_{i=1}^{N} P^{\max }(i)$.

\section{Computing the Optimal Policy}

Solving the dual problem (11) yields the optimal dual variable vector $\vec{\beta}^{*}$ and extremal network power vectors $\mathbf{P}^{*, \mathbf{i}}$, such that

$$
\begin{aligned}
g\left(\vec{\beta}^{*}\right) & =\min \{g(\vec{\beta}): \vec{\beta} \geq 0\} \\
& =V\left(\vec{P}^{*, i}, \vec{C}, \vec{X}\left(\vec{P}^{*, i}\right)\right) .
\end{aligned}
$$

The vectors $\mathbf{P}^{*, \mathbf{i}}$ are extreme points of $S_{P}$ that represent optimal transmission modes and the power levels of the transmitters in those transmission modes. In other words, assuming $H(\vec{C})$ is finite, an optimal schedule of network power vectors exists that consists solely of these extremal network power vectors $\mathbf{P}^{*, \mathbf{i}}$. This means that in the optimal policies we find, in each slot, every node is either not transmitting at all, or is transmitting at the maximum possible peak power to exactly one receiver. Moreover, since a hyperplane in $\left(L_{\mathcal{E}}+1\right)$ dimensional Euclidean space is determined by $L_{\mathcal{E}}+1$ linearly independent points contained within it, an optimal policy can be constructed that consists of at most $L_{\mathcal{E}}+1$ extremal network power vectors $\mathbf{P}^{*, \mathbf{i}}$. Let $K$ be the number of extremal network power vectors $\mathbf{P}^{*, \mathbf{i}}$ such that (15) holds. Define $\hat{K}=\min \left\{K, L_{\mathcal{E}}+1\right\}$. We thus have

$$
H(\vec{C})=g\left(\vec{\beta}^{*}\right)=V\left(\vec{P}^{*, i}, \vec{C}, \vec{X}\left(\vec{P}^{*, i}\right)\right),
$$


for all $i$ such that $1 \leq i \leq \hat{K}$.

It is easy to see that in any optimal policy (assuming $H(\vec{C})$ is finite), the average rate on each link $l, X^{a v g}(l)$, is exactly equal to $C(l)$. Otherwise, we could decrease the total average power further without violating any rate constraints thus contradicting the optimality of the solution. Let $X^{*}, i(l)$ denote the rate of link $l$ corresponding the optimal extremal network power vector $\mathbf{P}^{*}, \mathbf{i}$. Assuming $H(\vec{C})$ is finite, it can be shown that (12) guarantees the existence of a "weight vector" $\vec{\lambda}^{*}=\left\{\lambda^{*}(1), \lambda^{*}(2), \lambda^{*}(\hat{K})\right\}$ such that the equations (16) and (17) below are satisfied:

$$
\begin{aligned}
\sum_{i=1}^{\hat{K}} \lambda^{*}(i) X^{*, i}(l) & =C(l) \text { for all links } l \in \mathcal{E} \\
\sum_{i=1}^{\hat{K}} \lambda^{*}(i) & =1 \quad \text { where } \lambda^{*}(i) \geq 0
\end{aligned}
$$

Note that $\hat{K} \leq L_{\mathcal{E}}+1$, so the above system of equations is always overdetermined. There must be $\hat{K}$ of these equations which are linearly independent. Once we have identified such a set of equations, we solve them by inverting a $\hat{K} \times \hat{K}$ matrix.

The value of $\lambda^{*}(i)$ indicates the relative frequency at which the extremal network power vector $\mathbf{P}^{*, \mathbf{i}}$ is utilized in an optimal policy. This then determines a family of optimal policies, which can be constructed by alternating between the extremal network power vectors in accordance with the weight vector $\vec{\lambda}^{*}$. A specific optimal policy, for example, can be constructed using a psuedo-random number generator to select which extremal network power vector is used in each slot, such that the network power vector $\mathbf{P}^{*, \mathbf{i}}$ is used with probability $\lambda^{*}(i)$. Alternatively, a specific optimal schedule can be constructed in such a way as to provide "smooth service" to each link, though this topic is beyond the scope of the present paper.

\section{Reducing Complexity}

The algorithm developed to solve the dual problem considers all $M$ transmission modes. However, in the optimal schedule we have no more than $L_{\mathcal{E}}+1$ transmission modes. Since $M$ can be as large as $2^{L_{\mathcal{E}}}$, the complexity of minimizing the dual objective function can be exponential. Thus, reducing the set of possible transmission modes considered can greatly reduce complexity. A natural consequence of our algorithm for a network of nodes with omni-directional antennas is that, no node transmits or receives data at the same time in the optimal schedule due to high self interference. This observation can reduce the number of candidate transmission modes significantly. Typical wireless devices (e.g. laptops equipped with IEEE 802.11b wireless cards) are capable of decoding exactly one transmission at a time. For multihop networks comprising of such wireless devices, we could further reduce the candidate set of transmission modes by eliminating those transmission modes which consist of multiple simultaneous transmissions to a receiver. In general, in many cases it is possible using heuristics to eliminate many "obviously inefficient" transmission nodes from consideration, since they are unlikely to be used in an optimal policy. This idea is similar in spirit to the approach used in [6] to construct a set of "valid" links. The resulting policies we find can be shown to be optimal among all policies which are constrained to use only the set of chosen candidate transmission modes.

\section{E. Hierarchical Link Scheduling and Power Control}

The complexity of our algorithm taking into account some of the aforementioned simplifications is reasonable for a small number of links (about 15), but increases greatly even with the addition of an extra link. In [5], we present an hierarchical approach to link scheduling and power control to minimize the total average transmission power of all the links in a network with a large number of links. Each link in the network is subject to a prespecified average data rate requirement and each node is subject to a peak power constraint (identical to the constraints in this paper).

In this approach, links in the network are partitioned into groups called clusters. Each cluster is constrained to accomodate a limited number of links (e.g. at most 15). Furthermore, links in a cluster are constrained to be geographically close to each other. Links in one cluster are scheduled somewhat independently of links in other clusters, in that inter-cluster interference is modeled as static ambient noise. At the top level, scheduling is done at the cluster level to determine which clusters are active in each slot. The clusters that are simultaneously active coordinate to solve a global fixed point equation which determines all inter-cluster interference. This approach is scalable in the sense that clusters that are geographically remote need only a loose coordination with each other. Since clusters that are geographically far away from each other impose negligible mutual interference, we can activate a large number of clusters simultaneously. In fact, if the desired data rate on links are sufficiently low, the optimal policy activates all the clusters in the network simultaneously [5].

\section{Routing AND CAPACITY Allocation}

In the previous section, we presented an algorithm that finds an optimal link scheduling and power control policy to support a given traffic rate on each link in the network. In this section, we build on this to construct a policy which allocates traffic rates on each link in accordance with a routing algorithm, given a traffic matrix which describes the rate at which traffic is to be carried between specific source-destination pairs. The routing algorithm is guided by the computation of the minimum cost associated with optimal scheduling and power control for supporting traffic rates on each link that have already been allocated, as described in the previous section.

For example, consider a series of iterations, where at a given iteration we wish to support a small amount of additional traffic from one of the given source-destination pairs. Initially, no traffic is supported, i.e. the required minimum data rate on each link is zero. We compute the optimal values for the dual variables, $\vec{\beta}^{*}$. Using (13), we can estimate the cost of supporting additional traffic on each link using a first order 
Taylor series approximation. For example, suppose we wish to evaluate a given route $r$. Let $\vec{r}$ be the $L_{\mathcal{E}}$ dimensional vector, such that the component $r(l)=1$ if link $l$ is included in the route $\vec{r}$, and $r(l)=0$ otherwise. If we route an additional $\epsilon$ units of traffic along route $r$, the required data rate vector will increase from $\vec{C}$ to $\vec{C}+\epsilon \vec{r}$. Using (13), the cost of supporting the additional $\epsilon$ units of traffic along route $r$ can be determined from the approximation

$$
H(\vec{C}+\epsilon \vec{r})=H(\vec{C})+\epsilon \sum_{l \in \mathcal{E}} \beta^{*}(l) r(l) .
$$

Therefore, in order to determine the best possible path along which an additional increment of traffic should be routed, we can use a shortest path algorithm (such as Dijkstra's algorithm or the Bellman-Ford algorithm), where we set the weight for link $l$ to the sensitivity value $\beta^{*}(l)$. After we have identified the shortest path, we attempt to support the additional traffic along this path. If the new value of $\vec{C}$ is infeasible, we either reduce the step-size $\epsilon$ until the additional traffic can be supported, try supporting additional traffic for another source-destination pair, or otherwise reach a prespecified termination condition.

Indeed, since $H(\cdot)$ is convex, we can use the optimal routing framework developed by Bertsekas et al. (e.g., see [3]). In this class of algorithms, any feasible routing is first chosen. By incrementally adjusting the routing in an iterative manner, flows are shifted towards shortest paths at each iteration, and eventually a globally optimum routing is obtained. There are many possible variations, for example regarding whether flow is shifted simultaneously for every source destination pair, or whether flow is shifted one at a time for each source destination pair. In the present context, we are faced with a more difficult problem, since apparently $H(\cdot)$ is not differentiable everywhere. In addition, since evaluating the function $H(\cdot)$ is not trivial, it is more difficult to ascertain whether a given routing is feasible, i.e. the capacity of a given link depends on the rate carried by other links. Nevertheless, our initial numerical experiments that are based on (13) and framework developed by Bertsekas et. al. suggest that such an approach may be quite useful in practice.

In the examples, we will present below, it is apparent that $H(\cdot)$ is piecewise linear, which results in "sharp corners". When we use the above algorithm, it is possible that the link sensitivities can change abruptly. This can be partially addressed by choosing the step size $\epsilon$ sufficiently small. We can also "backtrack" by re-adjusting the rate allocations after each iteration, if necessary, to shift traffic towards shorter paths.

As expected, since $H(\cdot)$ is convex, we find that $\beta^{*}(l)$ increases after we allocate additional traffic on link $l$. As a result of this, routes which initially appear unattractive sometimes become more attractive after traffic is added on other links. As we will see, this phenomenon can lead to traffic from the same source-destination pair being routed over multiple paths, which is somewhat counterintuitive since all links share a common frequency band.

We remark that the approach of using optimal values of the dual variables as link weights in shortest path routing has also been used before in the context of revenue maximization in wired networks, by Mitra et. al. [13]. This work focuses on the asymptotic behavior of the link sensitivities with respect to uniform increases in demand rates. The authors of this previous work also observed the optimality of route diversification for price elastic traffic associated with maximizing network revenue.

The potential energy efficiency of a given link can be evaluated, assuming that all other links are idle. For our model, the minimum transmission energy required per bit on a link $(i, j)$ is inversely proportional to $G(i, j)$. A minimum energy path is defined as a path which has the smallest possible energy required to transport a fixed amount of data, irregardless of the delay associated with transporting that data. Previous work on routing in wireless networks (for example see [16][15]) is focused on energy efficiency without regard to delay, i.e. such work proposes using minimum energy paths.

In our framework, we find that the paths over which traffic is allocated in some optimum routings does not always correspond to using minimum energy paths. However, when required data rates are sufficiently low, our routing framework will apparently always use minimum energy paths. This is illustrated by example in the next section.

\section{Resource Allocation Examples}

In this section, we illustrate the gains of using our wireless resource allocation framework through a series of network topologies. We focus on simple examples in an attempt to gain insight from optimal allocations.

\section{A. Example 1: String Topology}

A string topology consists of a row of nodes connected by means of directed links as shown in Figure 1. This topology represents a "transport" network carrying data over long distances. Node 1 is the source of data and node 5 is the sink. Each node in this example has an omnidirectional antenna, and all nodes have identical peak transmission power constraints of 1 Watt. The path-loss between nodes was modeled as an inverse square of the distance for all the examples in this paper, i.e. $G(i, j)=\frac{1}{d(i, j)^{2}}$. The ambient noise power at all the nodes is assumed to be constant. We compare the maximum throughput of the single session between node 1 to node 5 achieved by our link scheduling policy to a TDMA scheduling policy. The TDMA policy schedules exactly one link at a time, activating each link at maximum power for a fourth of the time. We plot the ratio of throughputs achieved by the our link scheduling algorithm to TDMA with increasing values of ambient noise in Figure 2. The ambient noise is normalized with respect to the peak received power (from a single transmitter) in the network and is scaled by $\log _{2}($.$) (for all graphs in this section).$ The maximum throughput achieved by our policy is higher than TDMA for all values of ambient noise. In low noise regimes, TDMA is a near-optimal policy. However, as the level of ambient noise at nodes increases, the gains in throughput of our policy increase significantly. The optimal policy schedules 


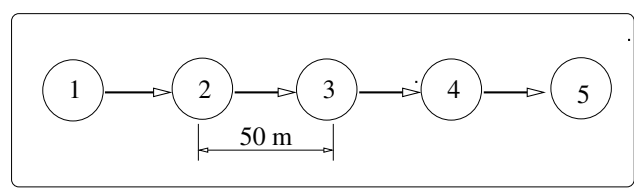

Fig. 1. String Topology

multiple transmissions at the same time, even in fairly low ambient noise regimes, thereby exploiting spatial reuse and thus outperforming TDMA. This effect is more pronounced in moderate and high ambient noise regimes where the optimal policy schedules concurrent transmissions even though they are in close geographic proximity.

To get a sense of how energy efficient the optimal policy is as it supports increasing traffic demands, we plot the total average power with increasing traffic rates for the session between node 1 and node 5 in Figure 3. For this experiment, we fix the level of the logarithm of the normalized value of ambient noise to -0.67 . This value corresponds to the $3^{\text {rd }}$ data point on the $\mathrm{x}$-axis in Figure 2. We also set $W^{\prime}=10^{7}$.

Figure 3 indicates that the total minimum average power is a linear function of the traffic demand for average rates below 4 Mbits/sec. Below 4 Mbits/sec, our scheduling policy reduces essentially to TDMA. Specifically, only one link is active at any time, each link is active for the same fraction and for some fraction of time all links are idle. As the traffic demand of the session increases beyond $4 \mathrm{Mbits} / \mathrm{sec}$, the total average power increases at a faster rate. For traffic load between $4 \mathrm{Mbits} / \mathrm{sec}$ and $4.98 \mathrm{Mbits} / \mathrm{sec}$, the transmission modes in the optimal policy are given by $[\{(1,2)\},\{(2,3)\},\{(3,4)\}$ and $\{(1,2),(4,5)\}]$. For traffic loads above $4.98 \mathrm{Mbits} / \mathrm{sec}$ to the maximum throughput possible, the optimal transmission modes are $[\{(1,2)\},\{(2,3)\},\{(3,4)\},\{(1,2),(3,4)\}$ and $\{(1,2),(4,5)\}]$. We see that in order to support high traffic loads, the optimal link scheduling policy activates a large number of links simultaneously. Note that the transmission modes in the optimal policy are half-duplex. Therefore, transmissions in the optimal schedule cannot consume a total average power of more than $2000 \mathrm{~mW}$ for this topology.

\section{B. Example 2: Diamond Topology}

In this experiment, we consider an asymmetric diamond topology as shown in Figure 4 to illustrate the substantial increase in throughput by splitting traffic over multiple routes including paths that are energy inefficient. Nodes in this topology are equipped with omnidirectional antennas, and the peak transmission power each node is fixed at 1 Watt. Node 1 is the only source of data and node 4 is the sink. We assume $G(1,4)=$ $\frac{1}{d(1,4)^{4}}$; the path loss between all other nodes is given by the inverse square law of distance. We consider our integrated routing and scheduling algorithm over this diamond topology, where all possible routes from the source to the destination are

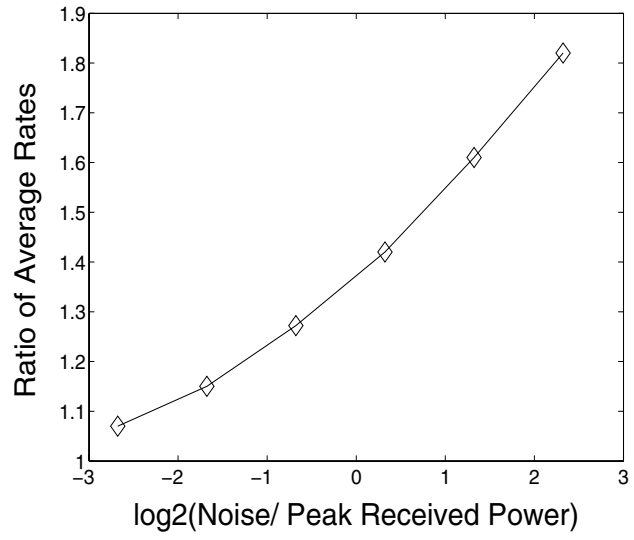

Fig. 2. String Topology: Ratio of maximum throughput for optimum scheduling Vs TDMA.

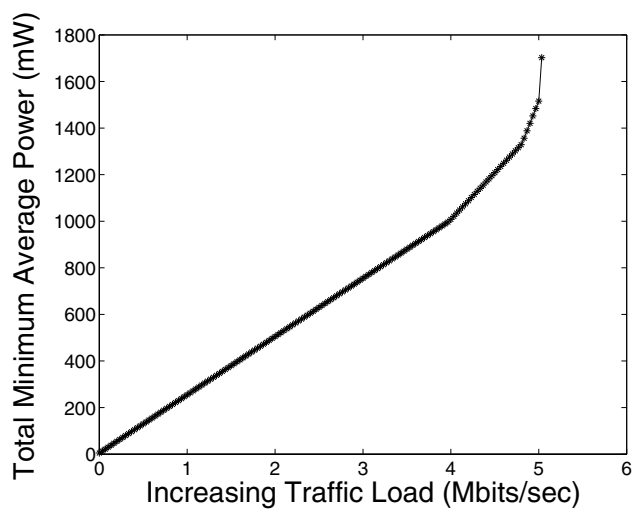

Fig. 3. String Topology: Total Minimum Average Power in milliwatts Vs Increasing traffic demands in Mbits/sec

allowed except for the single hop route $(1,4)$. For comparison purposes, we also considered our scheduling algorithm where only the links $(1,2)$, and $(2,4)$ are allowed to be used, which corresponds to routing over a single minimum energy path $1 \rightarrow 2 \rightarrow 4$.

We compare the ratio of throughputs for these polices in Figure 5. Clearly, using multiple paths yields higher throughputs for all values of ambient noise. The increase in throughput by splitting traffic over multiple paths is significant even for moderate levels of ambient noise. This result is somewhat surprising, since all links are sharing a common bandwidth and

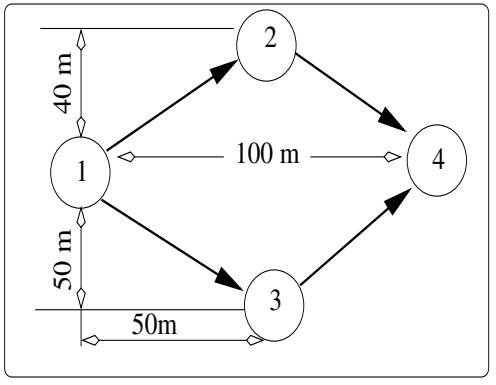

Fig. 4. Diamond Topology 


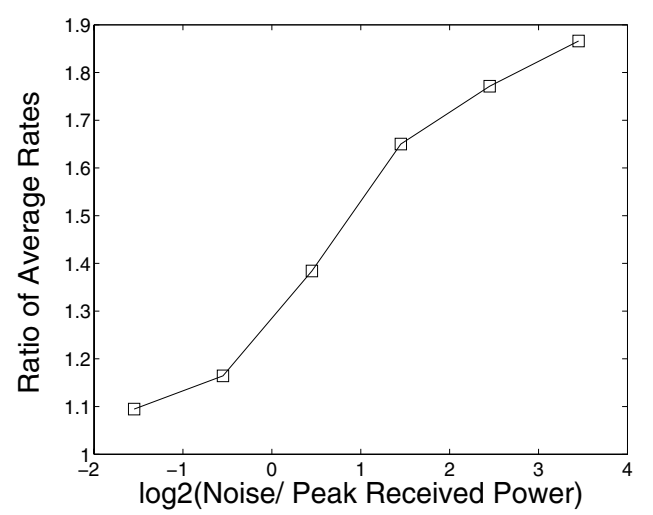

Fig. 5. Diamond Topology: Ratio of throughputs achieved by using multiple paths to using a single path $1 \rightarrow 2 \rightarrow 4$.

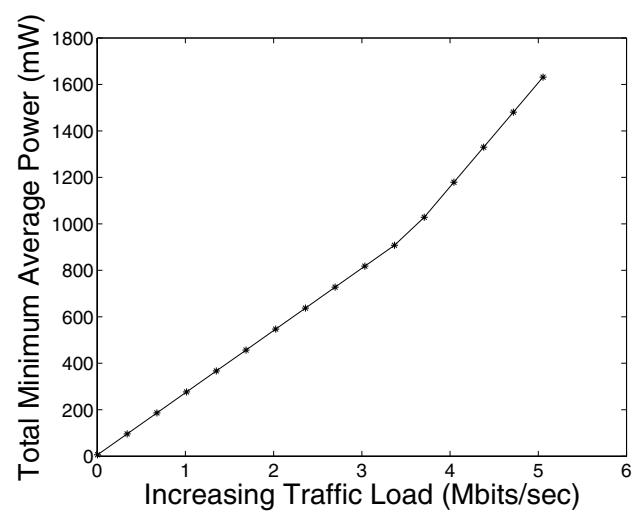

Fig. 6. Diamond Topology: Total Minimum Average Power in milliwatts Vs Increasing traffic demands in Mbits/sec

we have a per-node peak power constraint. An intuitive explanation of this is that by splitting the traffic over both paths, the transmission modes $\{(1,2),(3,4)\}$ and $\{(2,4),(1,3)\}$, which do not have much interference, can be alternated in time. This results in substantial delivery of data to the destination in every slot. If we only allow the path $1 \rightarrow 2 \rightarrow 4$, in order to avoid self interference, the links $(1,2)$ and $(2,4)$ must be active one at a time, so that node 4 will only be delivered data in every other slot.

To illustrate energy efficiency issues, we plot the total minimum average power with increasing traffic demand at node 1 in Figure 6. The logarithm of the normalized value of ambient noise for this point is 0.45 and corresponds to the $3^{\text {rd }}$ data point on the $\mathrm{x}$-axis in Figure 5. We set $W^{\prime}=10^{7}$. The maximum data rate achieved by TDMA using the minimum energy path $1 \rightarrow 2 \rightarrow 4$ is $3.65 \mathrm{Mbit} / \mathrm{sec}$. In comparison, our policy is capable of supporting a data rate of up to $5.05 \mathrm{Mbits} / \mathrm{sec}$ by using both the minimum energy path as well as the non-minimum energy path. For traffic loads below 3.65 Mbits/sec, our policy is essentially identical to TDMA over the path $1 \rightarrow 2 \rightarrow 4$. As the traffic load increases beyond $3.65 \mathrm{Mbits} / \mathrm{sec}$, the optimal policy uses starts using both paths by scheduling transmission modes $\{(1,2),(3,4)\}$

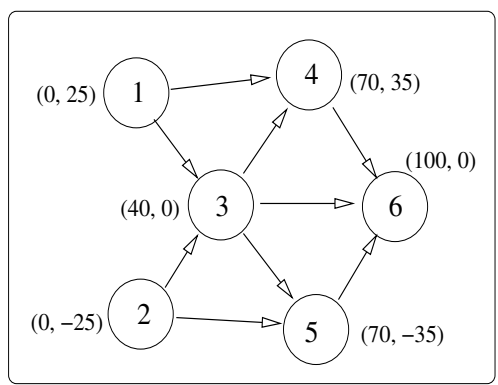

Fig. 7. Hierarchical Topology: Access Network

and $\{(1,3),(2,4)\}$ in addition to TDMA transmission modes. The average power required to achieve higher traffic demands beyond 3.65 Mbits/sec increases with a greater slope as can be seen in Figure 6. The maximum throughput of $5.05 \mathrm{Mbits} / \mathrm{sec}$ is achieved by splitting traffic by using both paths and scheduling transmission modes $\{(1,2),(3,4)\}$ and $\{(1,3),(2,4)\}$ for a dominant fraction of time and scheduling transmission modes $\{(1,2)\},\{(2,4)\}$ for the remaining time. The optimal policy transmits a fair amount of traffic on both available paths, but transmits a greater share of its traffic on its minimum energy path. We have noted a 10 to $20 \%$ reduction in the throughput gains of the optimal policy than what is shown in figure 5 for the case when $G(1,4)=\frac{1}{d(1,4)^{2}}$.

\section{Example 3: Hierarchical Topology}

A hierarchical topology as shown in Figure 7 represents a complex topology such as an access network, where the number of nodes in each tier decrease as we get closer to the access point node 6 . This experiment highlights the significant gains in throughput by jointly routing and scheduling to an alternative policy that optimally schedules links over pre-determined minimum energy routes. Nodes 1,2 and 3 are sources of data and node 6 is the sink. We assume $G(1,6)=\frac{1}{d(1,6)^{4}}$ and $G(2,6)=$ $\frac{1}{d(2,6)^{4}}$; the path loss between all other nodes is given by the inverse square law of distance. The minimum energy paths for the source nodes are: $\{1 \rightarrow 3 \rightarrow 6\},\{2 \rightarrow 3 \rightarrow 6\}$ and $\{3 \rightarrow 6\}$ respectively. Our integrated routing and scheduling algorithm is allowed to utilize all possible paths to route their data to node 6 . We constrain the rates of each source to be identical. We plot the ratio of the total maximum throughput of our routing policy to that achieved by using only the minimum energy paths, as a function of ambient noise in Figure 8.

We find that at low noise levels, our policy supports a throughput at least $30 \%$ more than the data rate achieved using minimum energy paths alone. As the level of ambient noise increases, our policy supports a significantly higher throughput. The base policy schedules links in a TDMA sequence for all values of ambient noise. The optimal policy schedules concurrent links even for low levels of ambient noise. In the optimal policy, nodes 1 and 2 route a significant amount of their traffic over non-minimum energy paths $1 \rightarrow 4 \rightarrow 6$ and 


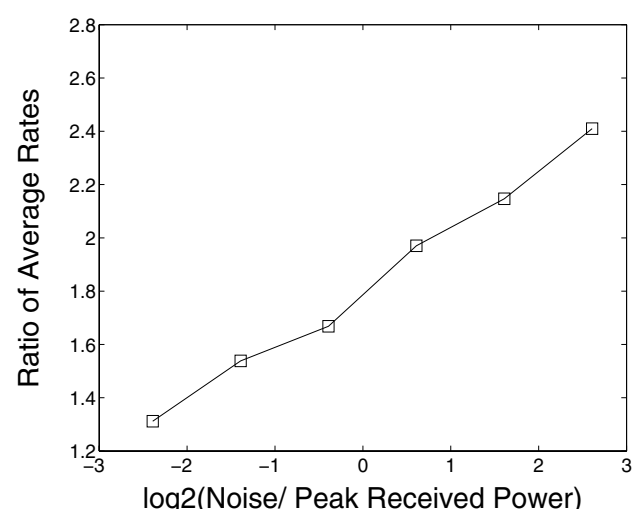

Fig. 8. Hierarchical Topology: Ratio of maximum throughputs achieved by using multiple paths to that using minimum energy paths only

$2 \rightarrow 5 \rightarrow 6$ respectively, while transmitting a relatively small fraction of their traffic over their minimum energy paths, for all values of ambient noise. For low ambient noise, the optimal policy schedules the transmission modes $\{(1,3)\},\{(2,3)\}$ and $\{(3,6)\}$ according to TDMA but also allows modes $\{(1,4),(5,6)\}$ and $\{(2,5),(4,6)\}$ to be active for reasonable fractions of time. As the level of ambient noise increases, the optimal policy splits node 3 's traffic over paths $3 \rightarrow 4 \rightarrow 6$ and $3 \rightarrow 5 \rightarrow 6$. In fact, for sufficiently high ambient noise, the optimal policy schedules a large number of concurrent links, the dominant transmission modes being $\{(1,4),(5,6),(2,3)\}$ and $\{(2,5),(4,6),(1,3)\}$ and $\{(1,4),(2,5),(3,6)\}$.

\section{DISCUSSION}

We have found that it is easy to generalize our problem formulation in a number of directions. For instance, we could minimize the total average transmitter and receiver energy required to achieve the target capacity on links in the network. This is discussed in the following subsection. Another constraint we could impose in our problem formulation (9) is a maximum peak power constraint for an arbitrary collection of nodes, rather than per each node. This may be useful to limit signal emissions from an entire network or subnetwork.

\section{A. Minimizing Total Average Transmitter and Receiver Power}

The total energy required to transmit a packet over a slot is the energy expended in the electronic circuitry to transmit at the desired power level [12]. The efficiency of power amplifiers is typically around $10 \%$ and therefore, the total power consumption at node $T(l)$ link is roughly $10 P(l)$. [12] notes that the energy involved in coding (e.g. convolution coding) packets is typically negligible.

The energy required to receive a packet of information is comprised of two components. The first component is the electronic energy required to operate PLL's, registers etc. and this is proportional to the data rate of the link. The second component is the energy required to decode the packet. While the decoding energy increases greatly as the SINR of the link decreases, this component of energy can be substantially lowered (by orders of magnitude) by using Application Specific Integrated Circuits to decode the received signal.

We therefore ignore the decoding energy component and coarsely model the power expended by a receiver $R(l)$ as a linear function of the data rate of link $l$. Let $P_{R}(l)$ denote the power expended by node $R(l)$ to receive data at rate $X(l)$. Then $P_{R}(l)=\theta(l) X(l)$ where $\theta(l)$ is the energy expended per bit that is constant for each receiver node $R(l)$. The new cost function that we would like to minimize is

$$
h\left(\vec{P}^{a v g}\right)=\sum_{l \in \mathcal{E}} \alpha(l) P^{a v g}(l)+\sum_{l \in \mathcal{E}} \theta(l) X^{a v g}(l)
$$

where $h\left(\vec{P}^{a v g}\right)$ is the sum total of average transmission and receiver power. The dual objective function corresponding to the new primal function (18) would have an additional average receiver power term. The dual problem corresponding to the new primal problem is similar to (11) except that without loss of generality, we can constrain $\beta(l) \geq \theta(l)$ for each link $l \in \mathcal{E}$. This constraint, $\beta(l) \geq \theta(l)$, allows us to exploit the coordinate concavity property and use the link scheduling and power control algorithms originally developed to solve problem (11), for the new dual problem.

Accounting for receiver power in our formulation changes the choice of routes and the rate allocations on links in each route. The changes in the routing policy are more pronounced in a network where nodes are in fair proximity to each other. Using multiple hops in such topologies would consume higher receiver energy than using a single hop, making multihop routing inefficient. In such topologies, ignoring receiver energy consumption in the problem formulation would have yielded multihop routing as the optimal routing policy.

Our integrated routing, scheduling and power control framework is well suited for slow fading wireless channels which are relatively constant for long durations of time. Outdoor wireless channels with strong line-of-sight paths typically tend to have a very stable channel strength and could be approximated as a constant for a time interval (block fading model) ranging from a few to several minutes. By periodically measuring the channel and accounting for the changing traffic requirements for each source-destination pair, we can compute the optimal routing, scheduling and power control policy and adapt it to the changing network conditions.

\section{CONCLUSION}

We have developed an integrated routing, link scheduling and power allocation policy for a general multihop network that minimizes the total average power consumption to support minimum average rate requirements per link. Our policy can support higher throughputs than with conventional approaches to radio resource allocation, at the expense of decreased energy efficiency. Our policy requires time synchronization between transmitters, and requires that channel conditions remain constant over several time slots. It appears to be most appropriate 
in the context of providing a means for wireless interconnection of fixed stationary nodes, such as wireless access nodes.

We find that our optimal power control policies are such that each node is either transmitting at peak power to a single receiver or not transmitting at all, which makes it simpler to implement. This also means that nodes can go to "sleep" much of the time, when they are not transmitting or receiving, which potentially conserves a great deal of energy [4]. The optimal link schedule time-shares a small number of optimal subsets of links $\left(\leq L_{\mathcal{E}}+1\right)$ in order to achieve the required data rates. The collection of optimal transmission modes depend on the level of ambient noise and the capacity allocations on links. The optimal policy for low required data rates or in low ambient noise regimes schedules links in a TDMA sequence. In moderate or high noise regimes, or to achieve higher data rates, the optimal strategy involves scheduling multiple simultaneous transmissions even though they may be in close geographic proximity.

A byproduct of solving the scheduling and power control problem yields link sensitivities that tell us the change in the minimal total average power with respect to a perturbation of the required data rate on a link. The minimal required average power is a convex function of the required data rates on each link. This leads us to a routing algorithm that is based on previously developed non-linear programming techniques.

We find that using minimum-energy paths to route traffic and TDMA to schedule links is near-optimal in low noise regimes and in the case where the required average data rates are small. As the level of ambient noise increases, or the required average data rates increase, our optimal routing policy exploits the use of multiple paths to support higher data rates, and the optimal scheduling policy makes use of concurrent transmissions on links. We find that non-minimum energy paths can be exploited to increase throughput, despite the fact that all links share a common bandwidth.

\section{REFERENCES}

[1] N. Bambos, S. Chen and G. Pottie, "Radio Link Admission Algorithm for Wireless Networks with Power Control and Active Link Quality Protection”, Proc. IEEE Infocom'95, pp. 97-104, 1995.

[2] D. Bertsekas, "Non-linear Programming", Athena Scientific, 1999.

[3] D. Bertsekas and J. Tsitsiklis, "Parallel and Distributed Computation: Numerical Methods", Prentice Hall 1989.

[4] B. Chen, K. Jamieson, R. Morrison and H. Balakrishnan, "Span: An Energy-Efficient Coordination Algorithm for Topology Maintenance in Ad Hoc Wireless Networks ", Proc. ACM MOBICOM Conference, July 2001.

[5] R. L. Cruz and A. V. Santhanam, "Hierarchical Link Scheduling and Power Control in Multihop Wireless Networks," Allerton Conference 2002, Monticello IL, October 2002.

[6] T. Elbatt, A. Ephremides, "Joint Scheduling and Power Control for Wireless Ad-oc Networks", Proc. IEEE Infocom 2002.

[7] A. Ephremides, T. V. Truong, "Scheduling Broadcasts in multihop radio networks", IEEE Trans. on Communications v.38, Apr. 1990.

[8] G. Foschini, Z. Miljanic, " A Simple Distributed Autonomous Power Control Algorithm and its Convergence", IEEE Transactions on Vehicular Technology, vol. 42 no. 4, pp 641-646, Nov. 1993.

[9] P. Gupta, P. R. Kumar, "The Capacity of Wireless Networks", IEEE Transactions on Information Theory, vol. IT-46, no. 2, pp. 388-404, March 2000.
[10] L. Kleinrock and J. A. Silvester, "Optimum Transmission Radii in Packet Radio Networks or Why Six is a Magic Number", National Telecommunications Conference, Birmingham, Alabama, pp. 04.3.1-5, December 1978.

[11] H. Luo, S. Lu, V. Bharghavan, "A New Model for Packet Scheduling in Multihop Wireless Networks", ACM Mobicom, August 2000.

[12] R. Min and A. Chandrakasan, "A Framework for Energy-Scalable Communication in High-Density Wireless Networks", Proc. ISLPED '02, Monterey, 2002.

[13] D. Mitra, K.G.Ramakrishnan and Q. Wang, "Combined Economic Modeling and Traffic Engineering: Joint Optimization of Pricing and Routing in Multi-Service Networks,", Proc.17th International Teletraffic Congress 2001.

[14] T. S. Rappaport, "Wireless Communications: Principles and Practice", Prentice Hall.

[15] V. Rodoplu and Teresa Meng, "Minimum energy mobile wireless networks", Proc. ICC'98 vol. 3, pp. 1633-1639, June 1998.

[16] S. Singh, M. Woo and C. S. Raghavendra, "Power-aware routing in mobile ad hoc networks", Proc. of Fourth Annual ACM/IEEE Conference on Mobile Computing and Networking pp. 181-190, Oct. 1998.

[17] S. Toumpis, A. J. Goldsmith, "Capacity Regions for Wireless Adhoc Networks", International Symposium on Communication Theory and Applications, April 2001. 